\title{
EFFICACY OF DOUBLE-LUMEN INTUBATION PERFORMED BY PARAMEDICS ON PATIENTS WITH LUNG DAMAGE. EXPERIMENTAL, PILOT SIMULATION TRIAL
}

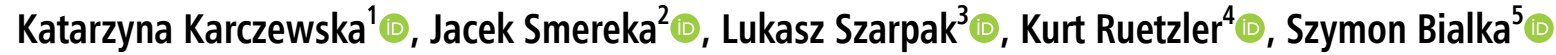 \\ 'Department of Anesthesiology, Mazovian Specialist Hospital, Radom, Poland \\ ${ }^{2}$ Department of Emergency Medical Service, Wroclaw Medical University, Wroclaw, Poland \\ ${ }^{3}$ Lazarski University, Warsaw, Poland \\ ${ }^{4}$ Departments of Outcomes Research and General Anesthesiology, Anesthesiology Institute, Cleveland Clinic, Cleveland, Ohio, United States \\ ${ }^{5}$ Department of Anaesthesiology and Intensive Care, Medical University of Silesia, Zabrze, Poland
}

\begin{abstract}
INTRODUCTION: The airway management and the implementation of optimal oxygen therapy in trauma patients, especially unconscious ones, is a key element of prehospital management. However, in cases of trauma lung or bronchial rupture, both lungs ventilation may not be advisable. In such cases, intubation with a double-lumen endotracheal tube may be helpful, allowing to provide one-lung ventilation. The aim of the experimental study was to assess the impact of short training in intubation using double-lumen tubes on the effectiveness of intubation performance and one-lung ventilation by paramedics in a simulated trauma patient setting.
\end{abstract}

MATERIAL AND METHODS: This was a prospective, observational, randomized experimental trial. The study involved 30 paramedics. The participants had to perform tracheal intubation using Double Lumen Tube VivaSight-DL (VS-DL; ETView Ltd.; Misgav, Israel) under normal airway conditions. The effectiveness of the first intubation attempt, the time of intubation and the Cormack-Lehane scale were evaluated.

RESULTS: The effectiveness of the first attempt at intubation with a double-lumen tube was $90 \%$ and the total effectiveness of intubation was $100 \%$. The median time of the procedure was 63 (IQR; 38-72.5) seconds.

CONCLUSIONS: In the simulation study, the paramedics were able to intubate using a double tube after a short training.

KEY WORDS: tracheal intubation; paramedic; one-lung ventilation; prehospital; medical simulation.

Disaster Emerg Med J 2020; 5(1): 7-11

\section{INTRODUCTION}

Airway management has always been one of the key elements of emergency procedures [1-3]. In many situations, including cardiopulmonary resuscitation, endotracheal intubation is recognized by such scientific societies as American Heart Association (AHA) or European Resuscitation Council (ERC) as the gold standard for airway management during resuscitation procedures $[4,5]$. It allows for complete separation of the airway from the external environment, which makes it possible to perform asynchronous resuscitation, use positive end-expiratory pressure or, in extreme situations, administer drugs with the use of endotracheal route. However, there are many 
cases in which both lungs ventilation may not be recommended or even adversely affect the patient's homeostasis. An example is a situation where there has been traumatic lung injury or bronchial rupture. Tracheobronchial injuries are rare events but often life-threatening [6]. In this case, ventilation, especially with positive end-expiratory pressure, may lead to pneumothorax. Then it may be helpful to secure the airways with an intubation tube and a bronchial blocker or intubation with a double light intubation tube and to implement one-lung ventilation $[7,8]$.

The aim of the study was to evaluate the effectiveness of double-lumen intubation and single-lung ventilation performed by paramedics under conditions of simulated lung injury.

\section{MATERIAL AND METHODS}

The survey was an experimental study and was designed as a prospective, randomized observational study. The study protocol was approved by the Institutional Review Board of the Polish Society for Disaster Medicine (Approval no. 42.02.2019.IRB). The study was conducted with the use of medical simulation techniques. The study involved 30 paramedics, who, before starting the study, expressed a written voluntary willingness to participate in the survey.

\section{Study design}

Prior to the study, all participants attended a 60-minute training course that included both the theoretical part of tracheal intubation, intubation training with the evaluated device, and a 20-minute practical training with the Airway Management Simulator (BT Inc., Tongil-ro, Republic of Korea).

Double Lumen Tube VivaSight-DL (VS-DL; ETView Ltd.; Misgav, Israel) was used in the study. This tube is a modern double-lumen endotracheal tube. However, its advantage over standard double light tubes lies in its built-in camera, which is connected to the monitor via a fibre optic cable. The camera is positioned between the distal ends of the tube, allowing the tube to be inserted, including the observation of the distal end of the tube entering the left bronchus under visual control (Fig. 1) - without the need for the use of intubation fiberscopes.

During the target study, participants were required to perform VS-DL endotracheal intubation under normal airway conditions in a patient requiring single lung ventilation. To insert the tube between the vocal folds, the participants used a la-

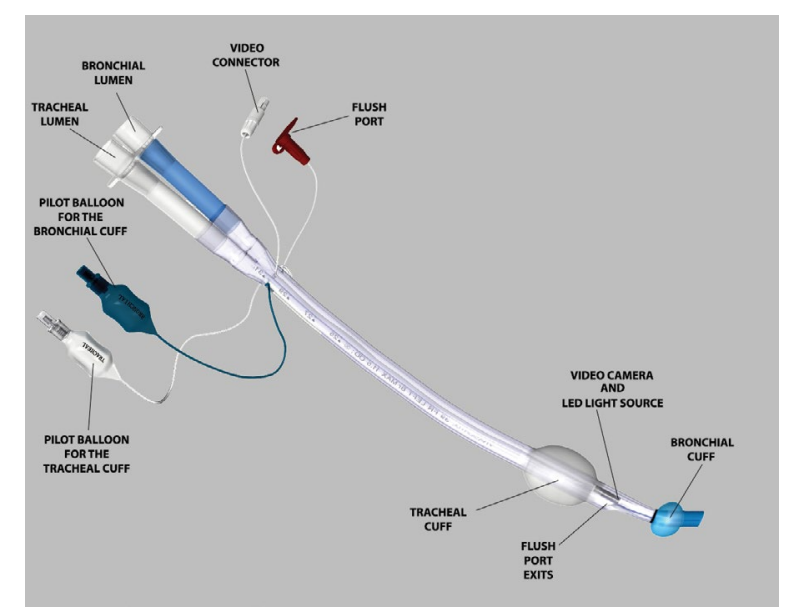

FIGURE 1. Double Lumen Tube VivaSight-DL

ryngoscope with a Macintosh blade (size 3; Heine Optotechnik, Munich, Germany). For this purpose, an adult SimMan 3G (Laerdal, Stavanger, Norway) simulator was used, which was placed on a standard stretcher to simulate pre-hospital conditions. The order of participants was random. During the study, only the intubator and researchers were present in the room and other participants were not allowed to stay in the room in order to reduce the risk of learning by observation.

\section{Measurements}

The primary endpoint was the rate of successful intubation. Successful intubation was defined when the one-lung of the simulator was inflated after the tracheal tube was connected to a self-inflating bag. Failed intubation was defined as an intubation time more than $120 \mathrm{~s}$ or when intubator gave up. The secondary endpoint were time to achieve successful DLT intubation, defined as the time when the laryngoscope passed the teeth to when the tip of the bronchial limen passed through the glottis, as confirmed visually by the operator using the video image. Moreover, we measure best view during laryngoscopy using the classification described by Cormack and Lehane [9].

\section{Simple size calculation}

The sample size was calculated with $G^{*}$ Power 3.1 with a two-tailed t-test (Cohen's $d=0.8$, alpha error $=0.05$, power $=0.95$ ). With the minimum of 30 participants necessary.

\section{Statistical analysis}

The Statistical Package Statistica 13.3EN (Tibco Inc., Tulusa, OK, USA) was used for statistical analysis. Re- 
sults obtained from each trial were compared using two-way repeated-measures analysis of variance for intubation time and Cormack-Lehane grade, and Fisher's exact test for success rate. Data are presented as median and interquartile range (IQR) or a number and mean percentage. $P<0.05$ was considered statistically significant.

\section{RESULTS}

\section{Participants characteristics}

The study involved 30 paramedics with more than 5 years of work experience. The median age of the study participants was 30.5 (IQR; 29-35) years. None of the participants in the study had previously had any experience in intubating with a double-lumen tube, but each had experience in intubating with a standard endotracheal tube.

\section{Intubation parameters}

The effectiveness of the first attempt at intubation with a double-lumen tube was $90 \%$ and the total effectiveness of intubation was $100 \%$. The median time of the procedure was 63 (IQR; 38-72.5) seconds. In the evaluation of the Cormack-Lehane scale, during the first phase of the insertion of a double-lumen tube between the vocal folds using a Macintosh-blade laryngoscope, $80 \%$ of the patients indicated the first degree of the Cormack-Lehane scale, while $20 \%$ of the patients indicated the second degree of the glottis visualization according to this scale.

\section{DISCUSSION}

Double-lumen intubation and one-lung ventilation are highly specialized procedures [10-12]. Due to the technique of this intubation as well as the higher costs of the equipment necessary to perform intubation compared to standard endotracheal tube, it is rarely used in pre-hospital conditions $\left[{ }^{3}\right]$. However, the development of medical technology and the development of video laryngoscopes and intubation tubes with built-in video circuits - including double-lumen tubes - may change this trend [13, 14]. There are many clinical situations in which it is reasonable to separate one lung from another and ventilate the other lung. Such clinical situations may include traumatic lung injury or bronchial rupture. Ventilation using both lungs may be ineffective and may also affect the hemodynamic parameters of the patient by inducing pneumothorax or pneumomediastinum. In such a case, the optimal solution may be double-lumen tube intubation or endotracheal intubation and the use of a bronchial blocker.

One-lung ventilation, using a double-lumen endotracheal tube or bronchial blockers is an important technique used in various clinical scenarios, but requiring more technical skills. The skills of physicians' non-experts in double-lumen endobronchial tube and bronchial blocker insertion decreases within two months without practice $\left[{ }^{15}\right]$ but this procedure can be standardized and taught efficiently [16]. Several training modules and simulation training were proposed to improve knowledge and skills in bronchial blocker placement and one-lung ventilation for anaesthesiologists [17]. Some airway simulators have been proposed for lung isolation with double-lumen endotracheal tubes a bronchial blockers insertion training [18] as well as modifications to existing Airways management trainers [19].

In the author' study, the effectiveness of the first attempt of intubation with a double-lumen tube was $90 \%$ and the total effectiveness of intubation was $100 \%$. The results are similar to other studies where videolaryngoscopes where used to facilitate endotracheal intubation $[20,21]$. Bakshi et al. compared the time to intubation for double-lumen tube insertion using the McGrath ${ }^{8}$ MAC VL versus direct Macintosh laryngoscope and revealed that this parameter was similar on both groups, however videolaryngoscopy was associated with better glottis visualization, reduced need of external laryngeal manipulation and fewer complications [22].

In a prospective observational study performed in 26 academic and community hospitals, Langiano et al. revealed that malposition rate for double-lumen tube was $14 \%$ and for bronchial blockers was $33 \%$ but the frequency of bronchoscope use was unexpectedly low [23]. It should be noted that intubation with the use of Double Lumen Tube VivaSight-DL does not require additional equipment, which may not be available in prehospital conditions.

In the presented study, the median time of the procedure was 63 (IQR; 38-72.5) seconds. Chang et al. compared GlideScope and lighted stylet for double-lumen endobronchial tube intubation in terms of intubation time, success rate of first attempt at intubation, difficulty in double-lumen tube advancement toward the glottis, and postoperative sore throat and hoarseness. The intubation time 
for double-lumen tube was shorter in the lighted stylet group compared with the GlideScope group (30 [28-32] s vs. 45 [38-53] s). The success rate of DLT intubation in the first attempt $(96.9 \%$ vs. $90.6 \%$ for lighted stylet and GlideScope, respectively) [20]. Yoo et al. compared in an open-label, randomized controlled, non-inferiority trial the time to intubation over a fiberscope using a silicone left double-lumen tube and polyvinyl chloride single lumen tube. The median time to intubation over the fiberscope was $20 \mathrm{~s}$ in the double-lumen group and $23 \mathrm{~s}$ in the single-lumen tube group [24]. El-Tahan et al. compared that laryngoscopy using the Airtraq and King Vision vs. Macintosh or GlideScope laryngoscopes in terms of intubation times for successful double-lumen endobronchial tube intubation by users with mixed experience. The channelled Airtraq resulted in shorter times for achieving successful double-lumen tube intubation (21 s) comparing to GlideScope $57.5 \mathrm{~s}[21]$.

There are certain limitations in the study. The first one is to perform the study in the conditions of medical simulation, not in the real conditions of prehospital intubation, however, the choice of such a method was intentional and dictated by the fact that the use of medical simulators during the teaching and evaluation of medical procedures allows for full standardization of the conditions for the performance of the procedure, as well as does not entail the risk of complications in the real patient resulting from incorrectly performed intubation, excessive duration of the procedure or repeated procedure $[25,26]$. The second limitation is the fact that only paramedics are included in the study group, however, it was considered that this professional group will be the most optimal as a pilot group because paramedics relatively often meet the necessity of protecting the airway of trauma patients in pre-hospital settings [27]. The authors are currently investigating the extension of the study group to include physicians as well as other emergency scenarios, including difficult airway conditions.

Among the strengths of the study are, among others, the randomized character of the study as well as the use of one of the most modern double light tubes in the study.

\section{CONCLUSIONS}

In the simulation study, the paramedics were able to intubate using a double tube after a short training.

\section{REFERENCES}

1. Umobong EU, Mayo PH. Critical Care Airway Management. Crit Care Clin. 2018; 34(3): 313-324, doi: 10.1016/j.ccc.2018.03.006, indexed in Pubmed: 29907267.

2. Simma $L$, Cincotta $D$, Sabato $S$, et al. Airway emergencies presenting to the paediatric emergency department requiring advanced management techniques. Arch Dis Child. 2017; 102(9): 809-812, doi: 10.1136/archdischild-2016-311945, indexed in Pubmed: 28404553.

3. Szarpak L. Laryngoscopes for difficult airway scenarios: a comparison of the available devices. Expert Rev Med Devices. 2018; 15(9): 631-643, doi: 10.1080/17434440.2018.1511423, indexed in Pubmed: 30099914.

4. Link MS, Berkow LC, Kudenchuk PJ, et al. Part 7: Adult Advanced Cardiovascular Life Support: 2015 American Heart Association Guidelines Update for Cardiopulmonary Resuscitation and Emergency Cardiovascular Care. Circulation. 2015; 132(18 Suppl 2): S444-S464, doi: 10.1161/CIR.0000000000000261, indexed in Pubmed: 26472995.

5. Soar J, Nolan JP, Böttiger BW, et al. Adult advanced life support section Collaborators. European Resuscitation Council Guidelines for Resuscitation 2015: Section 3. Adult advanced life support. Resuscitation. 2015; 95: 100-147, doi: 10.1016/j.resuscitation.2015.07.016, indexed in Pubmed: 26477701.

6. Welter $S$, Hoffmann H. [Injuries to the tracheo-bronchial tree]. Zentralbl Chir. 2013; 138(1): 111-116, doi: 10.1055/s-0032-1328269, indexed in Pubmed: 23450402.

7. Galvagno SM, Nahmias JT, Young DA. Advanced Trauma Life Support Update 2019: Management and Applications for Adults and Special Populations. Anesthesiol Clin. 2019; 37(1): 13-32, doi: 10.1016/j. anclin.2018.09.009, indexed in Pubmed: 30711226.

8. ATLS Subcommittee, American College of Surgeons' Committee on Trauma, International ATLS working group. Advanced trauma life support (ATLS®): the ninth edition. J Trauma Acute Care Surg. 2013; 74(5): 1363-1366, doi: 10.1097/TA.0b013e31828b82f5, indexed in Pubmed: 23609291.

9. Glosser L. Assessment of endotracheal tube intubation. Review of existing scales. Disaster and Emergency Medicine Journal. 2017; 2(2): 91-93, doi: 10.5603/demj.2017.0017.

10. Levy-Faber D, Malyanker $Y$, Nir RR, et al. Comparison of VivaSight double-lumen tube with a conventional double-lumen tube in adult patients undergoing video-assisted thoracoscopic surgery. Anaesthesia. 2015; 70(11): 1259-1263, doi: 10.1111/anae.13177, indexed in Pubmed: 26192743.

11. Hung KC. Trachway video stylet use in double lumen tube insertion. Anaesthesia. 2015; 70(9): 1093-1094, doi: 10.1111/anae.13176, indexed in Pubmed: 26263859.

12. Palczynski P, Bialka S, Misiolek $H$, et al. Thyromental height test as a new method for prediction of difficult intubation with double lumen tube. PLoS One. 2018; 13(9): e0201944, doi: 10.1371/journal. pone.0201944, indexed in Pubmed: 30212462.

13. Templeton TW, Morris BN, Royster RL. The Video Double-Lumen Endobronchial Tube: Is the Benefit Worth the Cost? J Cardiothorac 
Vasc Anesth. 2018; 32(1): 275-276, doi: 10.1053/j.jvca.2017.06.021, indexed in Pubmed: 29102256.

14. Szarpak L, Kurowski A, Zaśko P, et al. Double-lumen tube tracheal intubation in a manikin model using the VivaSight Double Lumen: a randomized controlled comparison with the Macintosh laryngoscope. Am J Emerg Med. 2016; 34(1): 103-104, doi: 10.1016/j. ajem.2015.10.018, indexed in Pubmed: 26527175.

15. Latif RK, VanHorne EM, Kandadai SK, et al. Teaching basic lung isolation skills on human anatomy simulator: attainment and retention of lung isolation skills. BMC Anesthesiol. 2016; 16: 7, doi: 10.1186/ s12871-015-0169-7, indexed in Pubmed: 26790624.

16. Cerfolio RJ, Smood B, Ghanim A, et al. Decreasing Time to Place and Teach Double-Lumen Endotracheal Intubation: Engaging Anesthesia in Lean. Ann Thorac Surg. 2018; 106(5): 1512-1518, doi: 10.1016/j. athoracsur.2018.06.023, indexed in Pubmed: 30048631.

17. Foley ED, Odo N, Arthur ME. Developing Modules to Train Anesthesiology Residents \& Medical Students in a Lung Isolation Technique. J Educ Perioper Med. 2017; 19(2): E602, indexed in Pubmed: 28824935.

18. Failor $E$, Bowdle $A$, Jelacic $S$, et al. High-fidelity simulation of lung isolation with double-lumen endotracheal tubes and bronchial blockers in anesthesiology resident training. J Cardiothorac Vasc Anesth. 2014; 28(4): 865-869, doi: 10.1053/j.jvca.2013.07.015, indexed in Pubmed: 24231196.

19. Hirsch J, Generoso JR, Latoures R, et al. Simulation Manikin Modifications for High-Fidelity Training of Advanced Airway Procedures. A A Case Rep. 2016; 6(9): 268-271, doi: 10.1213/ XAA.0000000000000278, indexed in Pubmed: 26752178.

20. Chang JE, Kim H, Min SW, et al. A Randomized Controlled Trial Comparing the Utility of Lighted Stylet and GlideScope for Double-Lumen Endobronchial Intubation. J Cardiothorac Vasc Anesth. 2018; 32(1): 290-296, doi: 10.1053/j.jvca.2017.10.009, indexed in Pubmed: 29132804.
21. El-Tahan MR, Khidr AM, Gaarour IS, et al. A Comparison of 3 Videolaryngoscopes for Double-Lumen Tube Intubation in Humans by Users With Mixed Experience: A Randomized Controlled Study. J Cardiothorac Vasc Anesth. 2018; 32(1): 277-286, doi: 10.1053/j.jvca.2017.08.009, indexed in Pubmed: 29056498.

22. Bakshi SG, Gawri A, Divatia JV. McGrath MAC video laryngoscope versus direct laryngoscopy for the placement of double-lumen tubes: A randomised control trial. Indian J Anaesth. 2019; 63(6): 456-461, doi: 10.4103/ija.IJA_48_19, indexed in Pubmed: 31263297.

23. Langiano N, Fiorelli S, Deana C, et al. Airway management in anesthesia for thoracic surgery: a "real life" observational study. J Thorac Dis. 2019; 11(8): 3257-3269, doi: 10.21037/jtd.2019.08.57, indexed in Pubmed: 31559028.

24. Yoo JiY, Chae YJ, Park SY, et al. Time to tracheal intubation over a fibreoptic bronchoscope using a silicone left double-lumen endobronchial tube versus polyvinyl chloride single-lumen tube with bronchial blocker: a randomized controlled non-inferiority trial. J Thorac Dis. 2019; 11(3): 901-908, doi: 10.21037/jtd.2019.01.108, indexed in Pubmed: 31019779.

25. Abelsson A. Learning through simulation. Disaster and Emergency Medicine Journal. 2017; 2(3): 125-128, doi: 10.5603/ demj.2017.0027.

26. Frass M, Smereka J, Ruetzler K, et al. New Flexible Tip Bougie catheter for difficult airway intubation. A randomized, crossover pilot study. Disaster and Emergency Medicine Journal. 2019; 4(2): 50-54, doi: 10.5603/demj.2019.0010.

27. Truszewski Z, Czyzewski L, Smereka J, et al. Ability of paramedics to perform endotracheal intubation during continuous chest compressions: a randomized cadaver study comparing Pentax AWS and Macintosh laryngoscopes. Am J Emerg Med. 2016; 34(9): 1835-1839, doi: 10.1016/j.ajem.2016.06.054, indexed in Pubmed: 27369468. 